\title{
ANALYSIS OF EXCHANGE RATE PASS-THROUGH, FEAR OF FLOATING, AND IMPLEMENTATION OF INFLATION TARGETING FRAMEWORK
}

\author{
Agung Satryo, Lukytawati Anggraeni \\ Department of Economics Faculty of Economics and Management Bogor Agricultural \\ University (IPB), Kampus IPB Dramaga, Jl. Kamper, Bogor 16680, Indonesia
}

\begin{abstract}
This paper analyze the exchange rate pass-through and fear of floating behavior on 18 countries that adopting Inflation Targeting Framework (ITF). Vector Error Correction Model (VECM) is used: (1) to estimate the effect of exchange rate depreciation to inflation (passthrough); and (2) to examine the indication of fear of floating behavior. The result shows that passthrough effect has decreased in most countries after ITF where middle income countries have higher passthrough than high income countries. This effect did not disappear completely and still has a significant role to drive inflation. The interventions on exchange rate movement can be interpreted more as control of inflation than fear of floating. The implementation of ITF especially in middle income countries needs further to be reconsidered since it requires inflation as the only nominal anchor.
\end{abstract}

Keywords: Fear of floating, Inflation Targeting Framework, Passthrough, VECM

\section{INTRODUCTION}

Inflation Targeting Framework (ITF) is a new monetary framework which is mostly used by industrial countries at the begining, such as: New Zealand (1990), Canada (1991), United Kingdom (1992), Sweden (1993), and Australia (1993). The implementation of ITF in those developed countries lead to a positive effect towards the inflation and stability of economics where the inflation rate tends to be low and stable so it can increase the economic growth in those countries (Pohan, 2008).

The economic crisis in 1997/1998 which is mostly occured in developing countries brings a change on their exchange rate regime, from pegged or fixed exchange rate regimes to floating exchange rate regimes. In addition, there was a change in monetary framework from money targeting framework or exchange rate targeting framework to inflation targeting framework.

The impact of exchange rate movement towards the prices in a country, can be analyse by exchange rate passthrough (ERPT) which is define as percentage of the change of domestic price if domestic exchange rate changed by $1 \%$. Its impact to goods and services in a country can occured either directly (direct exchange rate passthrough) or indirectly (indirect exchange rate passthrough) (Majardi, 2000). 


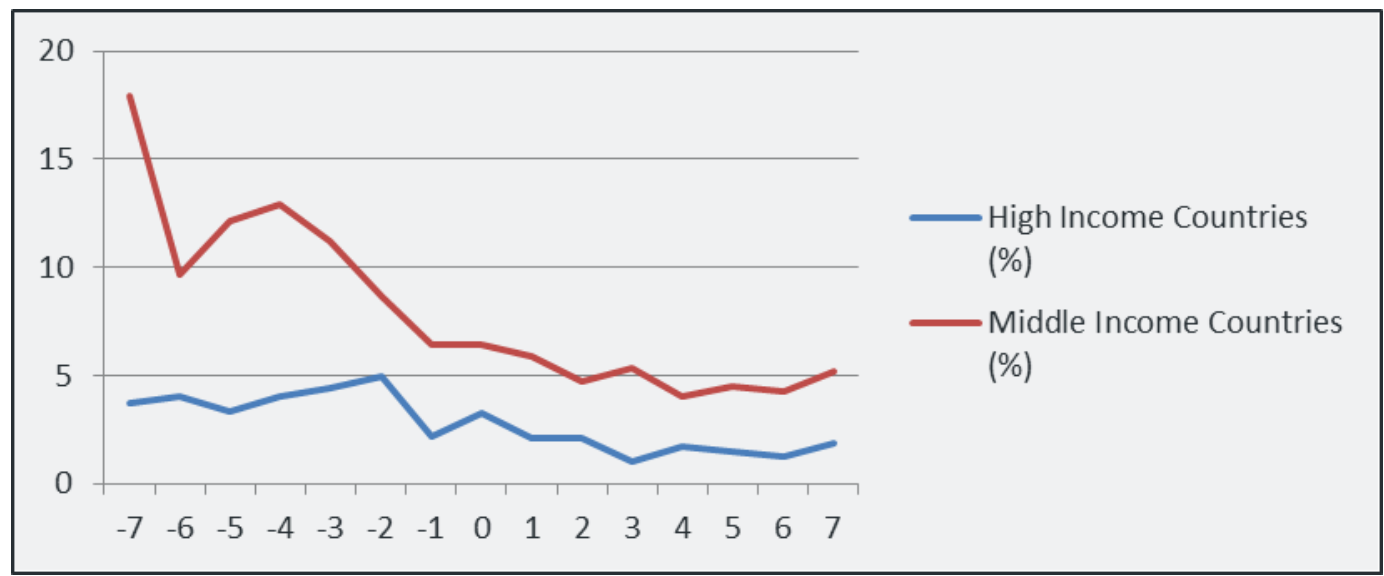

Source: Internasional Monetary Fund (2014)

Figure 1 The average of inflation rate in high income countries and middle income countries before and after the implementaton of ITF

Various studies have shown that after ITF, passthrough was still higher for middle income than high income countries. This shows that role of exchange rate movement affecting inflation is high particularly in middle income countries, thus makes the central bank more difficult to mantain inflation target. The implementation of fullpleged ITF makes the central bank should commit with attainment of inflation target that has been set before. In other side, the openness of economy that is getting large lately makes the movement of inflation always linked to exchange rate movement. The central bank can be trapped on exchange rate targeting and make it as nominal anchor instead of inflation targeting which should be the nominal anchor of ITF. This is often define as fear of floating (Nogueira, 2006).

There are vast literatures and studies about exchange rate passthrough in various countries, such as: Taylor (2000), Campa and Goldberg (2002), and Dilla (2014). However, the studies about comparison of the implementation of ITF and behaviour of fear of floating in high income countries and middle income countries is still rare. That is why this theme is important to understand the impact of the change on this framework.

\section{METHODS}

This study use time series and secondary data in 18 countries that adopting inflation targeting framework and divided into two groups: High income countries and middle income countries, in period before and after the implementation of ITF, so every country has different time periode between each other.

The data was collected from Internasional Financial Statistics (IFS), CEIC, dan OECD's iLibrary. Such as, Consumer Price Index (CPI), nominal exchange rate (domestic exchange rate/ US Dollar), interest rate (money market rate), and output (industrial production index). 
Table 1 Countries that adopting ITF

\begin{tabular}{lllll}
\hline \multirow{2}{*}{ No } & \multirow{2}{*}{ Countries } & \multirow{2}{*}{ Start Year } & \multicolumn{2}{c}{ Pre ITF } \\
\cline { 4 - 5 } \cline { 4 - 5 } 1. & & \multicolumn{2}{c}{ High Income Countries } \\
2. & Canada & 1991: M2 & 1984:M2-1991:M1 & 1991:M2-2014:M6 \\
3. & Sweden & 1993: M1 & 1986:M1-1992:M12 & 1993:M1-2014:M5 \\
4. & Norwich & 2001: M3 & 1994:M3-2001:M2 & 2001:M3-2009:M10 \\
5. & Switzerland & 2000: M1 & 1993:M1-1999:M12 & 2000:M1-2014:M6 \\
6. & South Korea & 2001: M1 & 1994:M1-2000:M12 & 2001:M1-2014:M5 \\
7. & Australia & 1993: M4 & 1984:M3-1993:M3 & 1993:M4-2013:M12 \\
8. & Finland & 1993: M1 & 1985:M3-1992:M12 & 1993:M1-1998:M12 \\
9. & Poland & 1998: M10 & 1991:M3-1998:M9 & 1998:M10-2014:M6 \\
10. & New Zealand & 1990: M1 & 1985:M1-1989:M12 & 1990:M1-2014:M3 \\
\hline & UK & 1992: M9 & 1988:M2-1992:M8 & 1992:M9-2014:M6 \\
11. & Indonesia & 2005: M7 & 1997:M7-2005:M6 & 2005:M7-2014:M5 \\
12. & Thailand & 2000: M5 & 1993:M5-2000:M4 & 2000:M5-2014:M7 \\
13. & Philipine & 2002: M1 & 1995:M1-2001:M12 & 2002:M1-2014:M6 \\
14. & Mexico & 2001: M1 & 1994:M1-2000:M12 & 2001:M1-2014:M7 \\
15. & South Africa & 2000: M2 & 1993:M2-2000:M1 & 2000:M2-2014:M6 \\
16. & Brazil & 1999: M6 & 1995:M1-1999:M5 & 1996:M6-2014:M6 \\
17. & India & 1990: M12 & 1982:M3-1990:M11 & 1990:M12-1998:M5 \\
18. & Peru & 2002: M1 & 1995:M10-2001:M12 & 2002:M1-2014:M4 \\
\hline
\end{tabular}

\section{Correction Model Analysis (VECM)}

Vector Error correction Model (VECM) is restricted VAR that used for unstationer variable at the level but have cointegration between them. This restriction should be given due to unstationer variable at level but have cointegration. Then, VECM use that cointegration restriction information into the spesification. VECM is often called VAR design for unstationer series that have cointegration relationship. Thus, there is a speed of adjustment from short term to long term in VECM (Firdaus, 2011).
The spesification of VECM model generally, as follows:

$$
\Delta y_{t}=\sum_{i=1}^{k-1} \Gamma_{k} \Delta y_{t-1}+\mu_{0}+\mu_{1} t+\alpha \beta y_{t-1}+\varepsilon_{t}
$$

Where $\mathrm{y}_{t}, \mu_{0 \mathrm{x}}, \mu_{1 \mathrm{x}}, \mathrm{t}, \Pi_{\mathrm{x}}, \mathrm{y}_{t-1}, \Gamma_{\mathrm{k}}, k-1$, $\varepsilon t$ are vector which contained of analysis variable, intercept vector, regression coefficient vector, time trend, $\alpha \times \beta$ where b' contained of long term cointegration equation, variable in-level, regression coefficient matrix, VECM order from VAR, and error term, respectively. 


\section{VECM Model}

$\left[\begin{array}{c}\Delta Y_{t} \\ \Delta N E R_{t} \\ \Delta I N F_{t} \\ \Delta R_{t} \\ \Delta i_{t}\end{array}\right]=\left[\begin{array}{lllll}\gamma_{11} & \gamma_{12} & \gamma_{13} & \gamma_{14} & \gamma_{15} \\ \gamma_{21} & \gamma_{22} & \gamma_{23} & \gamma_{24} & \gamma_{25} \\ \gamma_{31} & \gamma_{32} & \gamma_{33} & \gamma_{34} & \gamma_{35} \\ \gamma_{41} & \gamma_{42} & \gamma_{43} & \gamma_{44} & \gamma_{45} \\ \gamma_{51} & \gamma_{52} & \gamma_{53} & \gamma_{54} & \gamma_{55}\end{array}\right]\left[\begin{array}{c}\Delta Y_{t-1} \\ \Delta N E R_{t-1} \\ \Delta I N F_{t-1} \\ \Delta R_{t-1} \\ \Delta i_{t-1}\end{array}\right]+\left[\begin{array}{c}\varepsilon_{1 t} \\ \varepsilon_{2 t} \\ \varepsilon_{3 t} \\ \varepsilon_{4 t} \\ \varepsilon_{5 t}\end{array}\right]$

Where $Y_{t}, N_{E R}, I N F_{t}, R_{t}, i_{t}, \gamma_{t i j}$ , $\varepsilon_{i t}$, are Output, Exchange Rate (Domestic exchange rate/US Dollar, Inflation rate, International reserve, Money market rate, Coefficient of VECM Model regression, Error term, respectively.

Impulse response function (IRF) was used to examine the comparison of exchange rate passthrough among the countries before and after implementation of ITF. IRF is used to identify the responses of inflation rate toward shock of exchange rate. This value is the parameter of exchange rate passthrough.

Forecast error variance decomposition was used to see the impact of implementation of ITF toward monetary policy responses while there is a shock in exchange rate, hence we can obtain the analysis of fear of floating behavior. Based on Nogueira (2006), a country is indicated with fear of floating symptom if there is intervention to exchange rate either through international reserves or interest rate, meanwhile the exchange rate passthrough is considerably low. It makes the intervention of exchange rate cannot be linked as control of inflation.

\section{RESULT}

Comparison of exchange rate
passthrough before and after
implementation of ITF

Table 2 shows the exchange rate passthrough which is responses of inflation in 12 months to 1 percent exchange rate shock.

Table 2 Exchange Rate Passthrough

\begin{tabular}{|c|c|c|}
\hline Negara & Pre ITF & Post ITF \\
\hline \multicolumn{3}{|c|}{ High Income Countries } \\
\hline Canada & 0.196 & 0.007 \\
\hline Sweden & 0.006 & -0.001 \\
\hline Norwich & 0.003 & -0.009 \\
\hline Switzerland & -0.02 & 0.001 \\
\hline South Korea & 0.06 & 0.06 \\
\hline Australia & 0.023 & -0.028 \\
\hline Finland & 0.008 & 0.003 \\
\hline Poland & 0.024 & -0.008 \\
\hline New Zealand & 0.131 & -0.001 \\
\hline UK & 0.079 & -0.003 \\
\hline \multicolumn{3}{|c|}{ Middle Income Countries } \\
\hline Indonesia & 0.39 & 0.025 \\
\hline Thailand & 0.006 & -0.04 \\
\hline Philipine & 0.072 & -0.003 \\
\hline Mexico & 0.463 & 0.012 \\
\hline South Africa & 0.068 & 0.007 \\
\hline Brazil & 0.08 & 0.019 \\
\hline India & -0.076 & 0.099 \\
\hline Peru & 0.013 & 0.005 \\
\hline
\end{tabular}

Table 2 shows that there is decreasing of passthrough in most countries after implementation of ITF, where middle income countries still have higher passthrough than high income countries. New Zealand is noted as the most succesfull country in lowering passthrough in high income countries group from 0.131 to -0.001 after the implementation of ITF. South Korea has same passthrough before and after ITF. 
So does Switzerland which has low and constant passthrough for both periods.

The passthrough in middle income countries group is also decreasing, but the average is still higher than high income countries group. Indonesia, Mexico, Brazil, and India relatively have higher passthrough than other middle income countries after ITF. Thus, exchange rate is still important variable to drive domestic inflation.

\section{Fear of floating analysis}

Table 3 monetary policy responses analysis

\begin{tabular}{lll}
\hline \multicolumn{2}{l}{ No } & \multicolumn{2}{l}{ Countries } & \multicolumn{2}{l}{ Behavior } \\
1. & Cancome Countries & Fear of inflation \\
2. & Sweden & Fear of floating \\
3. & Norwich & Fear of inflation \\
4. & Switzerland & Fear of inflation \\
5. & South Korea & Fear of inflation \\
6. & Australia & Fear of floating \\
7. & Finland & Fear of inflation \\
8. & Poland & Fear of floating \\
9. & New Zealand & Fear of inflation \\
10. & UK & Fear of inflation \\
\hline Middle Income Countries \\
1. & Indonesia & Fear of inflation \\
2. & Thailand & Fear of floating \\
3. & Philipine & Fear of inflation \\
4. & Mexico & Fear of inflation \\
5. & South Africa & Fear of inflation \\
6. & Brazil & Fear of inflation \\
7. & India & Fear of inflation \\
8. & Peru & Fear of inflation \\
\hline
\end{tabular}

Forecast Error Variance Decomposition (FEVD) analysis shows that intervention on exchange rate either direct intervention by using international reserve instrument or indirect intervention by using interest rate instrument decreased in most countries after implementation of ITF. It is seen from decreasing of variance of international reserve and interest rate explained by variance of exchange rate.
Meanwhile, the variance of interest rate explained by variance of inflation increased after ITF. It means that most countries focused on inflation control instead of exchange rate control after ITF.

Considering that exchange rate shock still has effect on domestic inflation (exchange rate passthrough), intervention on exchange rate can be interpreted as control of inflation rather than fear of floating behavior. However, in this results, there are some countries with indication as fear of floating behavior. Countries with fear of floating symptom are Sweden, Australia, and Poland in high income countries group, and Thailand in middle income countries group.

The factors that makes a country has fear of floating behavior are: High foreign debt in foreign exchange rate; competitiveness effect; balance of payment effect (Ball and Reyes, 2004). Another factor that makes a country has fear of floating behavior is exchange rate fleksibility can increase uncertainty and decrease middle income countries access to international financial market.

\section{CONCLUSIONS}

In this paper we have analyse exchange rate passthrough and fear of floating behavior in high income and middle income countries before and after implementation of ITF. The results shows that exchange rate passthrough decreased after implementation of ITF in most countries. However, pass-through effect does not dissappear completely, thus exchange rate still has significant role to determine domestic inflation. FEVD analysis shows that exchange rate intervention decreased after ITF in most countries. Exchange rate shock towards inflation makes intervention on exchange rate can be interpreted as 
inflation control instead of fear of floating symptom.

The implementation of ITF as monetary framework needs to be reconsidered since it requires only inflation as nominal anchor. Efectivity of inflation targeting can decreased due to Exchange rate intervention. Passthrough effect is still exist after ITF particularly in middle income countries so that central bank should always make intervention on exchange rate to prevent domestic inflation caused by exchange rate shock. However, before central bank make the intervention, it needs to know clearly any factors that cause exchange rate depreciate. So it can make sure that it does not cause wrong policy respons.

\section{REFERENCES}

Ball C, Reyes J. 2004. Inflation Targeting or Fear of Floating in Disguise: The Case of Mexico. International Journal of Finance and Economics 9:49-69.

Berg C. 2000. Inflation Forecast Targeting: The Swedish Experience [Journal]. Stockholm (SE): Sveriges Riksbank Working Paper Series No. 100.
Campa G. 2006. Pass-through of exchange rates to consumption prices: What has changed and why. New York (US): Staff Report FederalReserve Bank of New York No. 261.

Dilla S. 2014. Exchange Rate Passtrough dalam Konteks Inflation Targeting Framework:

Kasus Empiris di 19 Negara di Dunia. Bogor: IPB pres.

Firdaus M. 2011. Aplikasi Ekonometrika untuk Data Paneldan Time Series. Bogor. IPB Pres.

Majardi F. 2000. Dampak Pass-through Pergerakan Nilai Tukar Rupiah Terhadap Laju Inflasi. Jakarta. Ocassional Paper Bank Indonesia.

Nogueira PR. 2006. Inflation Targeting, Exchange Rate Pass-trough and Fear of Floating [Journal]. Econstor.

Pohan A. 2008. Kerangka Kebijakan Moneter dan Implementasinya di Indonesia. Jakarta. PT Raja Grafindo Persada.

Taylor JB. 2000. Exchange Rate Passthrough Effects: A Dissaggregate Analysis Of Colombiaan Impor of Manufactured Goods. Economic Studies. Colombia. Department of the Central Bank of Colombia. 
Table 4 Variance of interest rate explained by variance of exchange rate

\begin{tabular}{|c|c|c|c|c|}
\hline Countries & Periods & 1 Month & 6 Month & 12 Month \\
\hline \multirow[t]{2}{*}{ Canada } & Pre ITF & 7.81645 & 27.54942 & 30.20365 \\
\hline & Post ITF & 1.071463 & 4.846230 & 5.211894 \\
\hline \multirow[t]{2}{*}{ Sweden } & Pre ITF & 2.012271 & 3.85959 & 3.351868 \\
\hline & Post ITF & 0.390066 & 3.113577 & 4.048951 \\
\hline \multirow[t]{2}{*}{ Norwich } & Pre ITF & 0.781181 & 0.918619 & 0.910311 \\
\hline & Post ITF & 0.179247 & 8.311035 & 9.466322 \\
\hline \multirow[t]{2}{*}{ Switzerland } & Pre ITF & 11.66846 & 12.42228 & 12.016 \\
\hline & Post ITF & 0.247990 & 0.063325 & 0.125192 \\
\hline \multirow[t]{2}{*}{ South Korea } & Pre ITF & 14.32397 & 31.09765 & 28.15206 \\
\hline & Post ITF & 3.315464 & 9.997735 & 11.99188 \\
\hline \multirow[t]{2}{*}{ Australia } & Pre ITF & 0.564987 & 7.65411 & 8.109677 \\
\hline & Post ITF & 3.836598 & 22.8387 & 26.7924 \\
\hline \multirow[t]{2}{*}{ Finland } & Pre ITF & 4.257436 & 27.58337 & 22.47378 \\
\hline & Post ITF & 4.441817 & 7.682253 & 8.743921 \\
\hline \multirow[t]{2}{*}{ Poland } & Pre ITF & 0.653875 & 0.69058 & 0.34483 \\
\hline & Post ITF & 0.106431 & 1.398875 & 1.334197 \\
\hline \multirow[t]{2}{*}{ New Zealand } & Pre ITF & 0.995822 & 26.02252 & 36.48023 \\
\hline & Post ITF & 0.001585 & 0.100124 & 0.095735 \\
\hline \multirow[t]{2}{*}{ UK } & Pre ITF & 11.5008 & 8.630392 & 8.525447 \\
\hline & Post ITF & 0.579219 & 6.966029 & 7.781542 \\
\hline \multirow[t]{2}{*}{ Indonesia } & Pre ITF & 1.395129 & 37.75485 & 43.64112 \\
\hline & Post ITF & 0.245056 & 0.310012 & 0.269653 \\
\hline \multirow[t]{2}{*}{ Thailand } & Pre ITF & 4.486496 & 5.317761 & 6.272180 \\
\hline & Post ITF & 3.489034 & 3.295796 & 3.412816 \\
\hline \multirow[t]{2}{*}{ Philipine } & Pre ITF & 0.236608 & 4.216861 & 4.47853 \\
\hline & Post ITF & 0.102992 & 0.203247 & 0.260488 \\
\hline \multirow[t]{2}{*}{ Mexico } & Pre ITF & 25.10861 & 54.858 & 57.37533 \\
\hline & Post ITF & 2.959431 & 7.413982 & 7.909458 \\
\hline \multirow[t]{2}{*}{ South Africa } & Pre ITF & 9.323478 & 29.40928 & 32.23603 \\
\hline & Post ITF & 1.071463 & 2.586435 & 2.753893 \\
\hline \multirow[t]{2}{*}{ Brazil } & Pre ITF & 0.853838 & 11.6407 & 12.32674 \\
\hline & Post ITF & 0.388557 & 3.502838 & 3.270021 \\
\hline \multirow[t]{2}{*}{ India } & Pre ITF & 2.387195 & 10.37858 & 13.69432 \\
\hline & Post ITF & 0.275673 & 0.447499 & 0.594767 \\
\hline \multirow[t]{2}{*}{ Peru } & Pre ITF & 0.012164 & 25.33358 & 20.22895 \\
\hline & Post ITF & 3.241689 & 8.458415 & 8.541282 \\
\hline
\end{tabular}

Notes: The number is percentage of variance of interest rate explained by variance of exchange rate after 1,6 , and 12 months 
Table 5 Variance of interest rate explained by variance of inflation

\begin{tabular}{|c|c|c|c|c|}
\hline Countries & Periods & 1 Month & 6 Month & 12 Month \\
\hline \multirow[t]{2}{*}{ Canada } & Pre ITF & 0.062416 & 0.01859 & 0.01177 \\
\hline & Post ITF & 10.03711 & 15.64934 & 17.3414 \\
\hline \multirow[t]{2}{*}{ Sweden } & Pre ITF & 0.634458 & 13.60431 & 26.18416 \\
\hline & Post ITF & 11.29097 & 7.725303 & 7.435974 \\
\hline \multirow[t]{2}{*}{ Norwich } & Pre ITF & 0.562069 & 0.545431 & 0.613425 \\
\hline & Post ITF & 0.222713 & 0.431723 & 0.372064 \\
\hline \multirow[t]{2}{*}{ Switzerland } & Pre ITF & 1.247193 & 6.92823 & 8.092511 \\
\hline & Post ITF & 0.540293 & 0.298175 & 0.281116 \\
\hline \multirow[t]{2}{*}{ South Korea } & Pre ITF & 4.974581 & 2.612050 & 1.933626 \\
\hline & Post ITF & 1.4517 & 8.276547 & 9.093486 \\
\hline \multirow[t]{2}{*}{ Australia } & Pre ITF & 1.457241 & 12.0587 & 14.52633 \\
\hline & Post ITF & 0.007375 & 0.025908 & 0.012695 \\
\hline \multirow[t]{2}{*}{ Finland } & Pre ITF & 0.276127 & 0.501099 & 2.405348 \\
\hline & Post ITF & 12.00577 & 29.45717 & 28.23065 \\
\hline \multirow[t]{2}{*}{ Poland } & Pre ITF & 5.275829 & 48.02014 & 60.49558 \\
\hline & Post ITF & 2.394815 & 10.00942 & 12.67453 \\
\hline \multirow[t]{2}{*}{ New Zealand } & Pre ITF & 0.63622 & 8.09879 & 11.39071 \\
\hline & Post ITF & 1.081848 & 8.464353 & 11.92389 \\
\hline \multirow[t]{2}{*}{ UK } & Pre ITF & 3.220354 & 1.259294 & 0.821387 \\
\hline & Post ITF & 0.014216 & 0.088645 & 0.060417 \\
\hline \multirow[t]{2}{*}{ Indonesia } & Pre ITF & 24.92612 & 32.47818 & 32.10813 \\
\hline & Post ITF & 3.428209 & 26.0166 & 29.43829 \\
\hline \multirow[t]{2}{*}{ Thailand } & Pre ITF & 0.403710 & 1.406325 & 0.712645 \\
\hline & Post ITF & 0.0786 & 1.404071 & 1.490215 \\
\hline \multirow[t]{2}{*}{ Philipine } & Pre ITF & 0.122975 & 9.796428 & 11.42763 \\
\hline & Post ITF & 0.315427 & 4.668927 & 8.043556 \\
\hline \multirow[t]{2}{*}{ Mexico } & Pre ITF & 18.94664 & 9.624112 & 8.759018 \\
\hline & Post ITF & 0.842923 & 4.304827 & 4.748631 \\
\hline \multirow[t]{2}{*}{ South Africa } & Pre ITF & 0.811872 & 4.192524 & 4.292827 \\
\hline & Post ITF & 10.03711 & 15.64934 & 17.3414 \\
\hline \multirow[t]{2}{*}{ Brazil } & Pre ITF & 6.738214 & 10.34006 & 13.50028 \\
\hline & Post ITF & 2.840434 & 17.98737 & 23.86888 \\
\hline \multirow[t]{2}{*}{ India } & Pre ITF & 7.847316 & 11.8941 & 12.71291 \\
\hline & Post ITF & 7.783034 & 2.610035 & 1.583121 \\
\hline \multirow[t]{2}{*}{ Peru } & Pre ITF & 0.034211 & 29.51039 & 43.51594 \\
\hline & Post ITF & 4.634863 & 12.0619 & 13.70073 \\
\hline
\end{tabular}

Notes: The number is percentage of variance of interest rate explained by varianceof inflation after 1,6 , and 12 months 
Table 6 Variance of international reserve explained by variance of exchange rate

\begin{tabular}{|c|c|c|c|c|}
\hline Countries & Periods & 1 Month & 6 Month & 12 Month \\
\hline \multirow[t]{2}{*}{ Canada } & Pre ITF & 13.23105 & 15.90569 & 16.99888 \\
\hline & Post ITF & 10.27830 & 8.249491 & 8.161157 \\
\hline \multirow[t]{2}{*}{ Sweden } & Pre ITF & 3.03057 & 3.83769 & 3.977690 \\
\hline & Post ITF & 3.066112 & 7.11541 & 7.925348 \\
\hline \multirow[t]{2}{*}{ Norwich } & Pre ITF & 3.591056 & 2.875646 & 2.676655 \\
\hline & Post ITF & 3.271937 & 1.667908 & 1.264868 \\
\hline \multirow[t]{2}{*}{ Switzerland } & Pre ITF & 3.354466 & 2.790583 & 2.956453 \\
\hline & Post ITF & 0.328963 & 0.048934 & 0.041512 \\
\hline \multirow[t]{2}{*}{ South Korea } & Pre ITF & 6.920251 & 1.491882 & 0.997184 \\
\hline & Post ITF & 25.67501 & 30.68508 & 30.58824 \\
\hline \multirow[t]{2}{*}{ Australia } & Pre ITF & 14.0071 & 20.30808 & 20.36699 \\
\hline & Post ITF & 2.808203 & 2.785207 & 2.306681 \\
\hline \multirow[t]{2}{*}{ Finland } & Pre ITF & 1.636326 & 4.903472 & 14.21598 \\
\hline & Post ITF & 1.306822 & 2.22855 & 2.614411 \\
\hline \multirow[t]{2}{*}{ Poland } & Pre ITF & 2.147112 & 2.087379 & 1.143587 \\
\hline & Post ITF & 18.91141 & 22.44574 & 22.28565 \\
\hline \multirow[t]{2}{*}{ New Zealand } & Pre ITF & 2.896759 & 1.849368 & 1.567321 \\
\hline & Post ITF & 1.58064 & 5.158534 & 5.397957 \\
\hline \multirow[t]{2}{*}{ UK } & Pre ITF & 48.10945 & 53.97087 & 55.4096 \\
\hline & Post ITF & 7.207862 & 6.451812 & 6.545576 \\
\hline \multirow[t]{2}{*}{ Indonesia } & Pre ITF & 0.082145 & 3.923148 & 3.670322 \\
\hline & Post ITF & 7.868739 & 7.8562 & 7.872501 \\
\hline \multirow[t]{2}{*}{ Thailand } & Pre ITF & 0.403978 & 7.809192 & 7.857587 \\
\hline & Post ITF & 2.626072 & 5.991780 & 6.301078 \\
\hline \multirow[t]{2}{*}{ Philipine } & Pre ITF & 2.145028 & 4.159129 & 4.217554 \\
\hline & Post ITF & 7.651996 & 17.8571 & 18.82021 \\
\hline \multirow[t]{2}{*}{ Mexico } & Pre ITF & 19.35459 & 14.60863 & 17.48311 \\
\hline & Post ITF & 10.28903 & 4.460832 & 3.373705 \\
\hline \multirow[t]{2}{*}{ South Africa } & Pre ITF & 14.74284 & 18.14091 & 20.12723 \\
\hline & Post ITF & 10.27830 & 8.249491 & 8.161157 \\
\hline \multirow[t]{2}{*}{ Brazil } & Pre ITF & 5.879066 & 7.801357 & 7.846514 \\
\hline & Post ITF & 1.456550 & 5.408142 & 6.253920 \\
\hline \multirow[t]{2}{*}{ India } & Pre ITF & 11.39702 & 27.62412 & 33.56837 \\
\hline & Post ITF & 0.047237 & 0.494937 & 0.717778 \\
\hline \multirow[t]{2}{*}{ Peru } & Pre ITF & 2.615471 & 0.568814 & 0.409158 \\
\hline & Post ITF & 1.574132 & 9.918944 & 11.26945 \\
\hline
\end{tabular}

Notes: The number is percentage of variance of international reserve explained by variance of exchange rate after 1,6, and 12 months 